\title{
Implementation of a Dual Mesh Method for Longitudinal Rolling in QForm V8
}

\author{
Dmitry Gerasimov ${ }^{1, a^{*}}$, Nikolay Biba ${ }^{2, b}$, Sergei Stebunov ${ }^{1, c}$, Maksim Kadach ${ }^{3, \mathrm{~d}}$ \\ ${ }^{1}$ QuantorForm Ltd., PO Box 74, 115088, Moscow, Russia \\ ${ }^{2}$ Micas Simulations Ltd., 107 Oxford Rd., Oxford, OX4 2ER, UK \\ ${ }^{3}$ National University of Science and Technology, Department of processing of metals pressure, \\ Leninskiy prospekt 4, 119049, Moscow, Russia \\ agerasimov@qform3d.com, ${ }^{b}$ nick@qform3d.com, cserg@qform3d.com, ${ }^{d}$ stendek89@yandex.ru
}

Keywords: Longitudinal rolling, simulation, QForm, dual mesh method, finite element mesh

\begin{abstract}
Numerical modeling of longitudinal rolling usually requires high computational power meanwhile it still takes long time to simulate multi pass process. Different methods for increasing of accuracy and speed of rolling simulation have being proposed recently. One of them is simultaneous use of two finite element meshes of different density, i.e. dual mesh method. The article is dedicated to solving of important problems of rolling technology and presents one example of rolling simulation in QForm V8 based on dual mesh method.
\end{abstract}

\section{Introduction}

The finite element simulation of longitudinal rolling processes needs special methods to deal with specific features of material flow and its interaction with tool. Software has not only to guarantee high accuracy of results and to be able to predict different defects, but also to provide reasonable solving time. A longitudinal rolling process is characterized by localized deformation zone, quasi steady-state material flow and by large part of the billet out of deformation zone. For this reason it makes sense to use special simulation methods. One of such methods is a dual mesh method $[1,2]$.

The presented paper describes an idea of dual mesh method as well as comparison of simulation results with experiments. The deformation of the material across the width is called spread. Spread is an important characteristic of longitudinal rolling and it depends on different process parameters such as grade of deformed material, rolling speed and roll diameter, friction conditions and elastic deformation of tools, cross section shape and the temperature of a rolled bar [3].

Numerical simulation helps to reduce the process design time and to choose the most optimal parameters of rolling for getting the necessary spread in all passes.

\section{Description of Simulation Method}

A comparison of effective strain distribution in longitudinal section of a rolled bar obtained by simulation with different densities of the finite element mesh is shown in Fig. 1. As can be expected the simulation with the finest mesh (adaptation parameter $a=5$ ) much more accurately represents the effective strain distribution.

Therefore, the mesh has to be fine not only in deformation zone but also in the whole volume of a work piece $[4,5]$. Otherwise a significant loss of accuracy inevitably happens particularly for the temperature gradients, accumulated effective strain and a product deformed shape. 


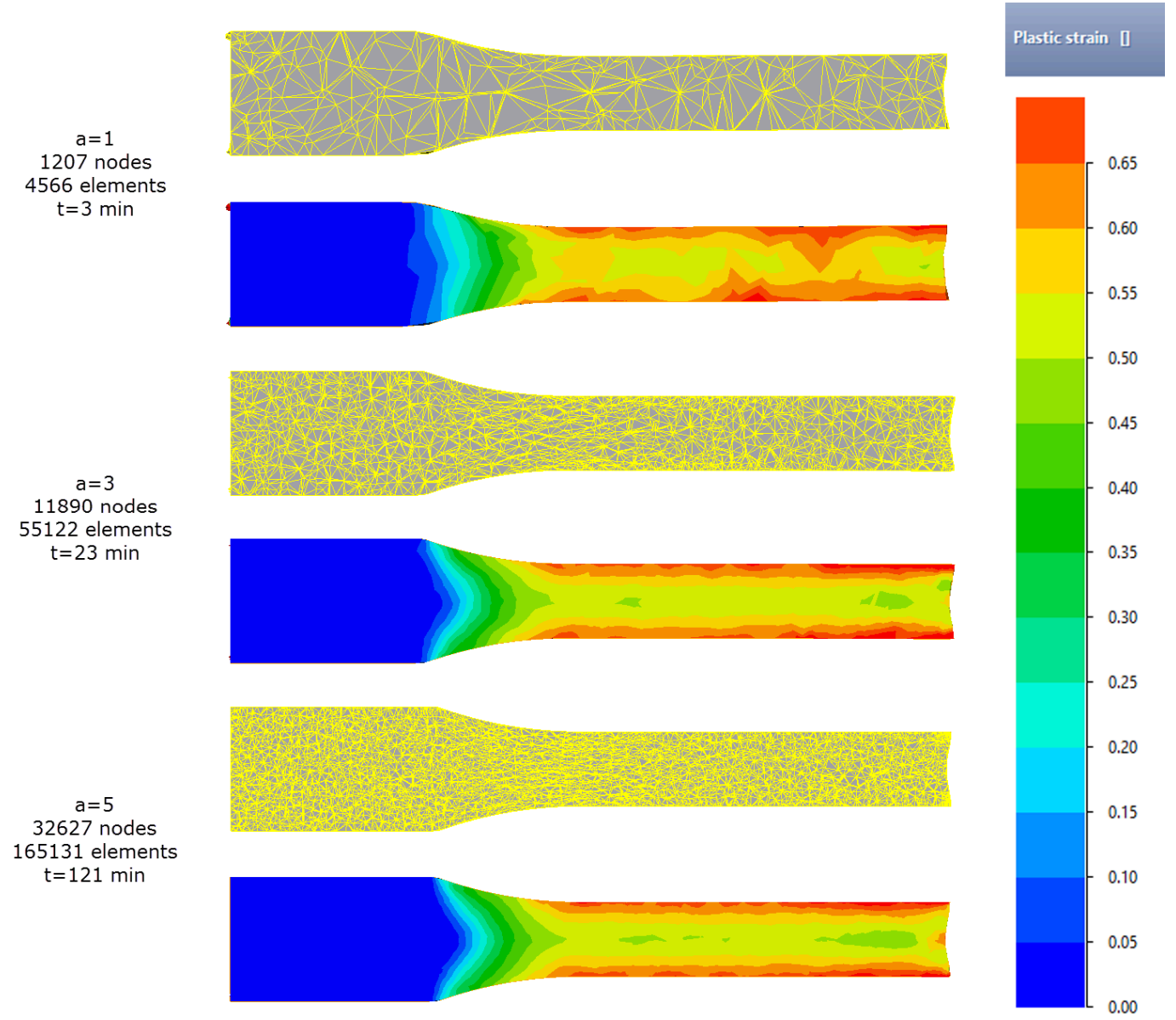

Fig. 1. Effective strain distribution in longitudinal section of rolled bar with different parameters of FE mesh density: $\mathrm{a}$ - mesh adaptation factor, $\mathrm{t}$ - simulation time of the whole process.

At the same time the fine finite element mesh in the whole product volume significantly slows down the simulation especially in case of 3D modelling. A dual mesh method is based on simultaneous use of two tetrahedral finite element meshes having different mesh density distribution in different parts of the product. The principle of a dual mesh method is shown in Fig. 2.
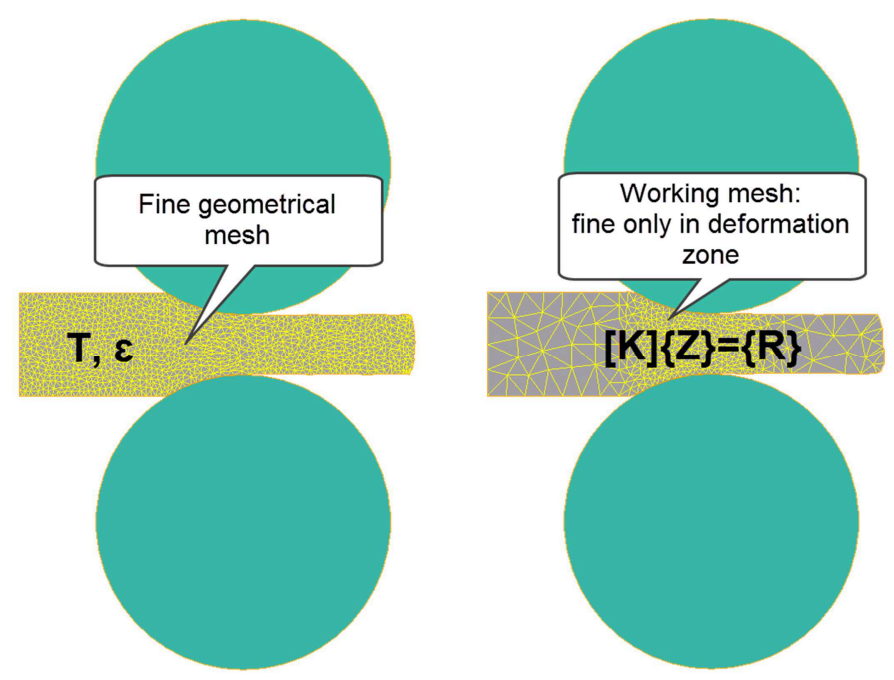

Fig. 2. Principle of a dual mesh method

The geometrical mesh is intended for an accurate description of a deformed body shape, solving a thermal problem and storing temperature and effective strain distributions. The geometrical mesh 
has to be very fine in the whole volume of a rolled product. The mechanical problem is simulated using another working mesh. The working mesh has to be fine only in a deformation zone. In areas of the product where the strain-rate is zero the working mesh can be very coarse. The results of deformation simulation obtained using working mesh are transferred to geometrical mesh for storing and using for simulation of coupled thermal problem. The densities of work and geometrical meshes are identical in the deformation zone. So, the data transfer of effective strain to the geometrical mesh is performed nearly without loss of accuracy.

Thus the dual mesh method allows using a very fine mesh for accurate description of geometry and thermal fields in the whole volume of a product without significant loss of simulation speed.

Special feature of the dual mesh technique implemented in software QForm V8 is a possibility to densify mesh automatically due to temperature, strain and flow velocities gradients.

\section{The Description of the Experiment}

A cold rolling of an aluminium round bar has been done in the laboratory. The experimental rolling mill is shown in Fig. 3. A rolling was performed in flat rolls with the diameter of $100 \mathrm{~mm}$ and the length of $80 \mathrm{~mm}$ and with the rotation speed of $60 \mathrm{rpm}$. The billet material is aluminium Al 99.5.

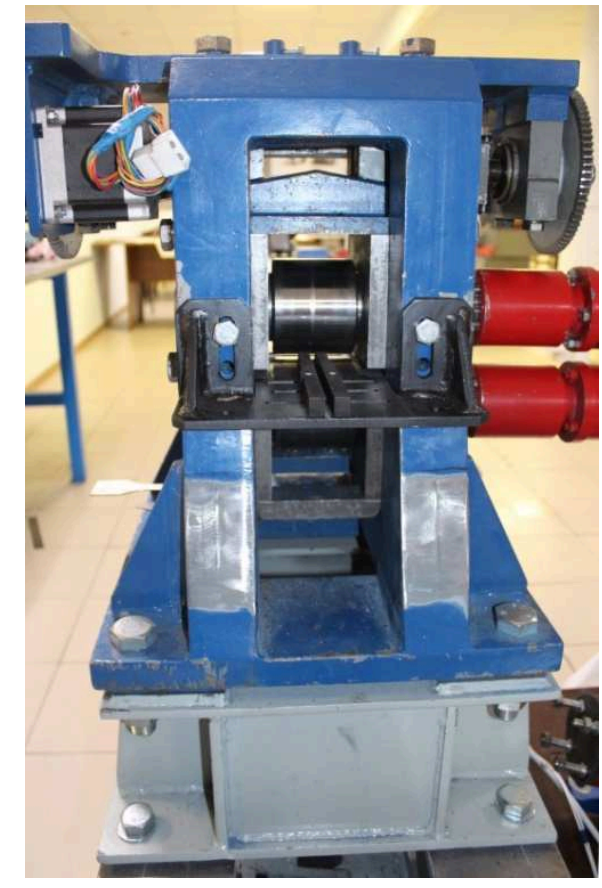

Fig. 3. Experimental equipment

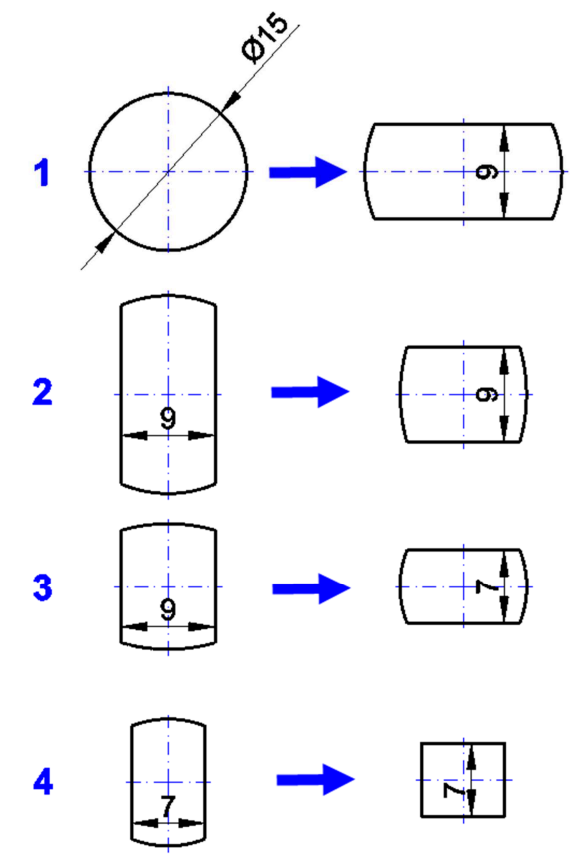

Fig. 4. Rolling passes of a round bar

A rolling of a round bar with the initial diameter of $15 \mathrm{~mm}$ to the height of $7 \mathrm{~mm}$ was done in 4 passes with the turnings by 90 degrees between the rolling passes. All passes are shown in Fig. 4 . The dimensions of a rolled bar in all 4 passes are shown in Table 1, where $h$ and $b$ are respectively the height and the width after each pass. Experimental samples after each pass are presented in Fig. 5. 
Table 1. Experimental results of the longitudinal rolling of the round bar, here $h$ and $b$ are the height and the width after each pass

\begin{tabular}{|c|c|c|c|c|}
\hline Pass & $\begin{array}{c}\mathrm{h}, \\
{[\mathrm{mm}]}\end{array}$ & $\begin{array}{c}\mathrm{b}, \\
{[\mathrm{mm}]}\end{array}$ & $\begin{array}{c}\Delta \mathrm{h}, \\
{[\mathrm{mm}]}\end{array}$ & $\begin{array}{c}\Delta \mathrm{b}, \\
{[\mathrm{mm}]}\end{array}$ \\
\hline 1 & 9.0 & 17.2 & 6.0 & 2.2 \\
\hline 2 & 9.0 & 10.8 & 8.2 & 1.8 \\
\hline 3 & 7.0 & 10.5 & 3.8 & 1.5 \\
\hline 4 & 7.0 & 8.0 & 3.5 & 1.0 \\
\hline
\end{tabular}

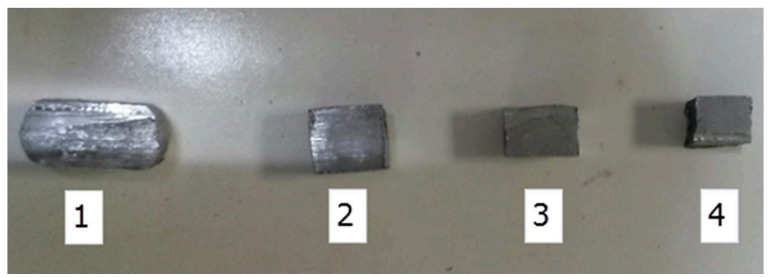

Fig. 5. Experimental samples after each pass

\section{The Simulation Results}

A cylinder with the length of $150 \mathrm{~mm}$ and the diameter of $15 \mathrm{~mm}$ was used as an initial workpiece for simulation. The deformed material was considered as visco-plastic continuum while the rolls were as rigid bodies. The properties of geometrical mesh are defined by an adaptation parameter that changes the sizes of all finite elements in inversed proportion. The adaptation of geometrical mesh was set equal to 5. The properties of a working mesh are defined by elements size $2.5 \mathrm{~mm}$ in the whole volume and $1 \mathrm{~mm}$ in the deformation zone.

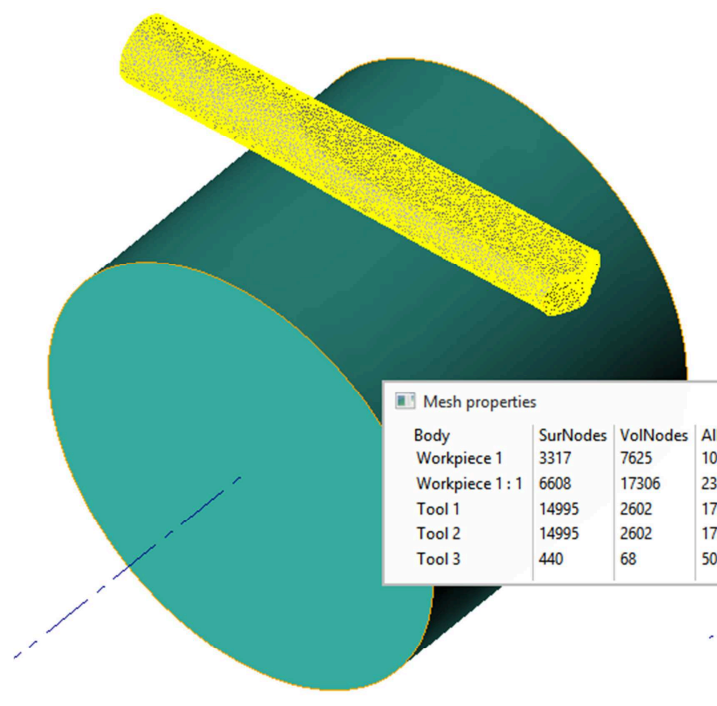

a

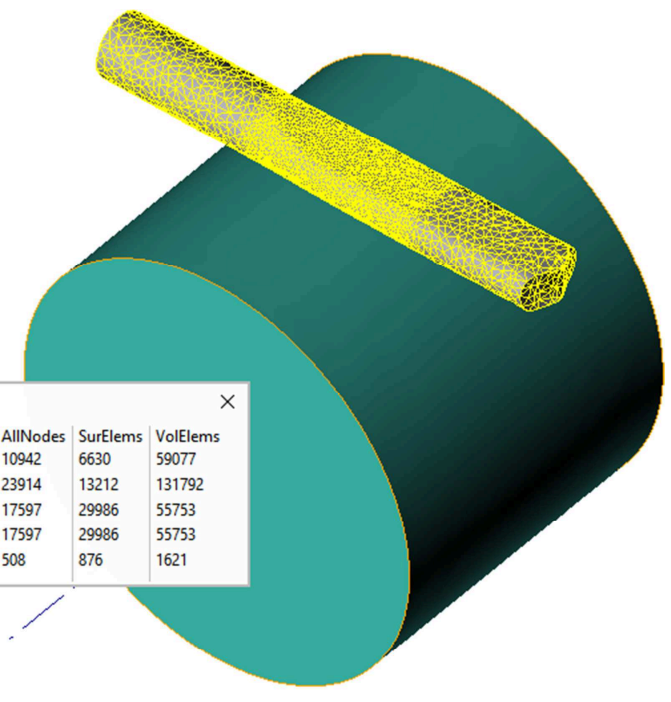

b

Fig. 6. Simulation of the first pass of longitudinal rolling: $a$ - geometrical mesh, $b$ - working mesh

For example, in the first rolling pass the working mesh has about 11000 nodes, and the geometrical mesh - about 24000 nodes. Both meshes are shown in Fig. 6. Simulation of the first pass using a dual mesh method lasts about 90 minutes. Simulation time of the same problem using the method with just one mesh having 24000 nodes was about 250 minutes. The effect of simulation speed increasing comparing to one mesh method is bigger in the case of longer product.

Simulation results when using lubrication friction coefficient $\mu=0.12$ as recommended in [6] are shown in Fig. 7. The experimental width values $b_{\text {exp }}$ are compared with simulated width $b_{\text {sim }}$ in Table 2. As seen, the simulation has shown the results very close to experiments. 
Table 2. Comparison of width after each pass by simulation in QForm V8 with experimental values: $\mathrm{h}$ - the height of the bar, $\Delta-$ the difference between the width in experiment $b_{\text {exp }}$ and in simulation $b_{\text {sim }}$

\begin{tabular}{|c|c|c|c|c|}
\hline Pass & $\begin{array}{c}\mathrm{h}, \\
{[\mathrm{mm}]}\end{array}$ & $\begin{array}{c}\mathrm{b}_{\text {exp }}, \\
{[\mathrm{mm}]}\end{array}$ & $\begin{array}{c}\mathrm{b}_{\text {sim, }} \\
{[\mathrm{mm}]}\end{array}$ & $\begin{array}{c}\Delta, \\
{[\%]}\end{array}$ \\
\hline 1 & 9.0 & 17.2 & 17.3 & 0.5 \\
\hline 2 & 9.0 & 10.8 & 11.3 & 4.6 \\
\hline 3 & 7.0 & 10.5 & 10.8 & 2.8 \\
\hline 4 & 7.0 & 8.0 & 8.3 & 3.8 \\
\hline
\end{tabular}

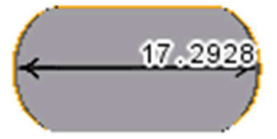

1

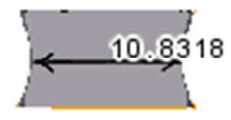

3

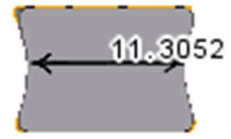

2

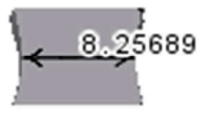

4

Fig. 7. Width of the workpiece after each pass obtained in simulation

\section{Summary}

To provide good simulation results in longitudinal rolling it is necessary to create a fine finite element mesh not only in the deformation zone but also in the whole volume of the product. The dual mesh method implemented in QForm V8 makes it possible to increase a speed of simulation several times in comparison with the standard single mesh method without loss of accuracy.

\section{References}

[1] N. Kim, S. Machida, S. Kobayashi, Ring rolling process simulation by the three dimensional finite element method, International Journal of Machine Tools and Manufacture, 30 (4), 1990, pp. 569-577

[2] Z. Hu, I. Pillinger, P. Hartley, S. McKenzie, P. Spence, Three-dimensional finite-element modelling of ring rolling, Journal of Materials Processing Technology, 45, 1994, pp. 143-148

[3] G.E. Dieter, H.A. Kuhn, S.L. Semiatin, Handbook of Workability and Process Design, American Society for Metals, Materials Park, 2003, pp. 233-238

[4] N. Biba, S. Stebunov, A. Vlasov, Material forming simulation environment based on QForm3D software system, Metal Forming 2008: Proc. of the 12th Int. Conf. On Metal Forming. Vol II, Ed. by M.Pietrzyk et.al. Krakow, Poland, 2008 - Verlag Stahleisen GmbH., pp. 611-616

[5] S. Stebunov, N. Biba, A. Vlasov, A. Maximov, Thermally and Mechanically Coupled Simulation of Metal Forming Processes, Proceedings of the 10th International Conference on Technology of Plasticity, Aachen, Germany, 2011, pp.171-175

[6] J.A. Schey, Metal Deformation Processes: Friction and Lubrication, Marcel Dekker, New York, 1970, p. 427 